\title{
In-Memory Policy Indexing for Policy Retrieval Points in Attribute-Based Access Control
}

\author{
Dominic Heutelbeck \\ dheutelbeck@ftk.de \\ FTK - Forschungsinstitut für \\ Telekommunikation und Kooperation \\ e.V. \\ Dortmund, Germany
}

\author{
Marc Lucas Baur \\ mlbaur@ftk.de \\ FTK - Forschungsinstitut für \\ Telekommunikation und Kooperation \\ e.V. \\ Dortmund, Germany
}

\author{
Martin Kluba \\ mkluba@ftk.de \\ FTK - Forschungsinstitut für \\ Telekommunikation und Kooperation \\ e.V. \\ Dortmund, Germany
}

\begin{abstract}
Attribute-Based Access Control (ABAC) systems are using machinereadable rules for making access control decisions. Rules are collected in documents, the named policies, or policy sets. These are expressed in a specific policy language, such as XACML, ALFA, or SAPL. Within systems implementing the ABAC reference architecture, policy documents are persisted in a Policy Retrieval Point (PRP). This paper addresses the problem of efficiently retrieving policy documents applicable to a given authorization request (or subscription) from the PRP. Applicability is determined by a specific section of the document, commonly named target expression. The target expression consists of matching conditions, more precisely Boolean expressions based on request (or subscription) data. This paper presents a novel in-memory data structure that is used to index policy documents. The index allows retrieving documents matching a given authorization request more efficiently from a large set of policies. The empirical evaluation demonstrates, that the proposed algorithm can reduce policy retrieval time in PRPs by up to $98 \%$, depending on the structure of the policies.
\end{abstract}

\section{CCS CONCEPTS}

- Security and privacy $\rightarrow$ Access control; Authorization; • Theory of computation $\rightarrow$ Sorting and searching.

\section{KEYWORDS}

access control, data structures, Boolean function search, policy languages, attribute-based access control

\section{ACM Reference Format:}

Dominic Heutelbeck, Marc Lucas Baur, and Martin Kluba. 2021. In-Memory Policy Indexing for Policy Retrieval Points in Attribute-Based Access Control. In Proceedings of the 26th ACM Symposium on Access Control Models and Technologies (SACMAT) (SACMAT'21), June 16-18, 2021, Virtual Event, Spain. ACM, New York, NY, USA, 12 pages. https://doi.org/10.1145/3450569.3463562

\section{INTRODUCTION}

Attribute-based access control (ABAC) [7] is a model for establishing authorization infrastructures across applications in complex

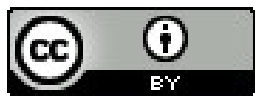

This work is licensed under a Creative Commons Attribution International 4.0 License.

SACMAT '21, June 16-18, 2021, Virtual Event, Spain.

(C) 2021 Copyright held by the owner/author(s).

ACM ISBN 978-1-4503-8365-3/21/06.

https://doi.org/10.1145/3450569.3463562 domains. In ABAC systems, access to resources is granted based on policies, i.e., rules and relationships determining which behavior of a user or process (the subject) is authorized when accessing different resources. The policies themselves are expressed using policy languages. The rules contained in the policies refer to traits, the so-called attributes, of relevant objects, such as name, date of birth, unique identifiers, location, or security clearance level. These attributes may refer to data contained in the decision request (or subscription) or may require accessing external data sources during policy evaluation to determine the outcome of the authorization process. These data sources are called Policy Information Points (PIPs). Other well-established access control schemes, such as Discretionary Access Control (DAC) [8], Mandatory Access Control (MAC) [8], and Role-Based Access Control (RBAC) [16] can be expressed by ABAC systems.

The leading standard for expressing ABAC policies is the eXtensible Access Control Markup Language (XACML) standard [19]. Further, ALFA [20] is a semantically identical simplified syntax for expressing XACML policies. A new policy language is SAPL [4] implements attribute stream-based access control (ASBAC) [3], an extension of $A B A C$ focusing on publish-subscribe communication models capable of processing streaming attributes (e.g., IoT data) and adds features beyond the expressiveness of XACML. Even though, in the case of SAPL a PDP processes decision subscriptions and not just decision requests, the problem discussed in this paper is independent of this difference. Thus, for simplicity, the remainder of the paper will only refer to requests. Typically a decision request is an XACML or JSON document containing a subject, action, resource, and environment object, expressing the authorization question: "May the subject do the action with the resource in the environment ?" These data objects may contain arbitrary XML or JSON data, depending on the application domain.

The functional architectures of XACML and SAPL share the concept of a policy enforcement point (PEP), which makes decision requests and enforces decisions made by a policy decision point $(P D P)$. Documents containing policies or policy sets are stored in a policy retrieval point $(P R P)$ as a collection of documents.

Whenever a PDP receives a decision request, it has to identify documents applicable to the request. Applicability is determined by the target element in XACML or a target expression in SAPL. Both are Boolean expressions on predicates of the request. In XACML these are enforced to be in Disjunctive Normal Form (DNF) and SAPL allows these to be expressed like in general-purpose programming languages without normalization. 
In Section 2.10 of the XACML standard [19], the process of identifying applicable policy statements is identified as policy indexing. This paper addresses the question of how efficiently perform policy indexing (also called policy retrieval). Note, that policy indexing is different from policy checking and evaluation. Evaluation occurs only after policy retrieval and proceeds to evaluate the retrieved applicable policies to determine a decision. The evaluation problem for ABAC policies is addressed by $[9,10,12]$. In addition, access to external Policy Information Points is limited to the policy body and its rules. Thus, target expressions are an important tool to minimize IO operations for efficient authorization decision making.

For policy indexing, XACML supports two approaches. Either to have the PDP form specific database queries or to load all available policies and individually check their target elements. The standard does not address how such queries could be formed and what the matching database scheme would look like. Thus, this paper uses the second approach as the base-line for evaluating the presented algorithm.

There are two conditions on target expressions to enable efficient indexing, as discussed in this paper. The predicates in the target expressions must only reference attributes contained in the decision request and not access external PIPs, and Boolean operators must be eagerly evaluating.

Policy indexing or retrieval can be reduced to the problem of searching in a multiset of Boolean expressions over predicates for elements that evaluate to true for a given decision request.

Indexing Disjunctive Normal Form (DNF) and Conjunctive Normal Form (CNF) Boolean expressions over a high-dimensional multivalued attribute space is examined by [18]. In this specific case, expressions contain predicates checking if a value is contained in a specific subset of potential values. The presented problem is to find expressions that are satisfied with a specific variable assignment. The proposed solution applies inverted lists, commonly used for ranked information retrieval, and partitions the conjunctions of size $k$ in a $K$-index.

The approach presented in $[14,15]$ limits Boolean expressions conjunctions over predicates, which allows space partitioning and space clustering approaches to building $B E-$ Trees.

Indexing of XACML policies is specifically addressed in [13] which creates an index consisting of a Matching-Tree and a CombiningTree. The Matching-Tree is built over the target elements, forming a decision diagram. In this graph, nodes represent the equality of attributes of a particular request. Leaf nodes are formed by Combining-Trees. A Combining-Tree is a tree of policies of each terminating node without their target sections. They are needed to preserve the tree structure and order of the policies, as otherwise, the results may differ. Support of incomplete lists of attributes and complex functions is added via the creation of special edges, that need separate evaluation. This may cause the evaluation to take multiple paths in the Matching-Tree and results in the combination of all Combining-Trees in their paths.

The Propagation algorithm as proposed by [2] shows a predicatebased approach inside a subscription environment. The idea is, to quickly compute the set of verified predicates of a request and use this result to access an attribute-clusters, linking predicates to subscriptions.
Quickly solving the target expressions of policies and policy sets requires an indexing algorithm, capable of optimizing any given target expression, including complex function evaluation and a re-indexing after policy changes. The constraints to the sets of functions imposed on the predicates by existing algorithms are used for optimization and limit their applicability to policies. The considering $[14,15]$ the BE-Tree approach is a promising candidate for indexing Boolean functions. While it cannot be transferred to the presented use-case without modification, it presents a valid option for indexing policies by target expressions.

All the presented approaches have some major or minor limitations with regards to their application to target expressions.

The main contributions of this paper are the proposal and evaluation of a novel index and search algorithm for indexing Boolean expressions and its application and evaluation in the domain of access control for policy indexing in ABAC systems like XACML or SAPL.

The paper is structured as follows. Section 2 will present a more refined problem statement. Section 3 index and retrieval algorithm. The algorithms correctness is proven and its runtime is analyzed. Also, the problem of identifying a good order in which predicates are to be evaluated is discussed. Section 4 presents the evaluation experiments using fully randomly generated policies or on randomly scaled up real-world policies. The paper concludes and describes possible future work in Sections 5 and 6.

\section{PROBLEM STATEMENT}

This paper examines policy indexing for ABAC policy languages like XACML and SAPL as defined in the introduction.

For all examples, generating policies and evaluation, SAPL is used. It offers a more compact and easier to read syntax compared to XACML.

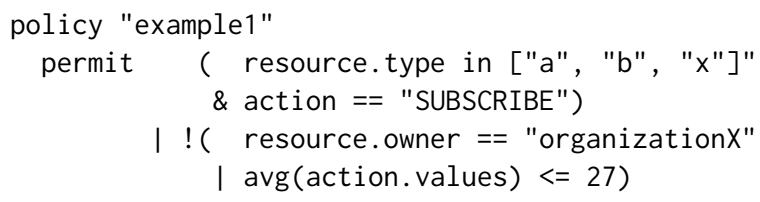

In this SAPL policy, the Boolean target expression is between the keywords permit and where, followed by additional rules. The evaluation of these rules is out of the scope of this paper. Only target expressions are examined.

In this example, the predicates for a decision request $s$ of the target expressions are:

- $p_{1}(s)=$ resource.type in ["a", "b", "x"]",

- $p_{2}(s)=$ action == "SUBSCRIBE",

- $p_{3}(s)=$ resource. owner == "organizationX"

- $p_{4}(s)=\operatorname{avg}($ action.values $)<=27$.

Thus, the target expression of the policy can be written as a Boolean expression using these predicates as follows:

$$
\left(p_{1}(s) \wedge p_{2}(s)\right) \vee \neg\left(\left(p_{3}(s) \vee p_{4}(s)\right)\right) .
$$

Similar Boolean expressions can be expressed in XACML target elements. Compared to the problems addressed by the approaches 
of $[14,15,18]$, the inner expressions in SAPL can contain more complex predicates compared to XACML (e.g., complex JSON queries and custom functions) and the policy indexing approach should be applicable to both. This flexibility has two computational drawbacks. Inner expressions may have significant evaluation costs associated with them (i.e., geometric operations for validating if the subject is located inside a geofence). The transformation of Boolean expressions into DNF may add cost with regards to the number of inner expressions/literals [11]. As in typical policies, the number of inner expressions in target expressions is very low, and updates to the index are a rare operation in comparison to the queries, this is unlikely to have a significant impact in the presented application domain.

The problem for both, XACML and SAPL, is the search in a multiset of Boolean expressions over predicates for elements that evaluate to true for a given decision request, i.e. the search for applicable policies. This paper examines:

a) Identifying in-memory index structures to be used by a PRP in an ABAC system based on XACML or SAPL.

b) How to use the index structures to efficiently retrieve applicable policies using the index?

c) How to deal with the predicate ordering problem implied by the proposed algorithm?

d) How does the algorithm perform compared to the naive strategy laid out by XACML?

e) How does the algorithm perform given worst-case random data compared to structured data.

\section{IN-MEMORY POLICY INDEX}

This section first defines several index structures and introduces a policy retrieval algorithm using these structures to identify applicable policies. Then the correctness of the algorithm is proven and its runtime is analyzed. Finally, the problem of identifying a good predicate order for the algorithm is discussed.

\subsection{Index Construction and Search Algorithm}

Before index construction, all target expressions are normalized to disjunctive normal form (DNF) with the minimal number of predicates in each conjunction. The predicates are identified as explained in the last section, which will in practice depend on the ABAC language used. During normalization, some formulas may be identified which are always satisfied. These indicate policies that are applicable to all requests and are stored outside of the main index to be included in any result.

Indexing and search are based on counting and elimination, attempting to avoid the evaluation of predicates and conjunctions when previously evaluated predicates allow to infer that the outcome of these predicates and conjunctions will not change the search result. The predicates are used as keys in the index data structure and each predicate is uniquely identifiable (e.g., by an integer identifier). Each conjunction can be identified as a set of predicates and can be indexed separately. Equally normalized formulas in DNF can be expressed as sets of conjunctions.

The basic idea of the algorithm is to iterate over the predicates in a precalculated order. Determining this order is the subject of question c) of Section 2, which is answered in Section 3.4.
Whenever a predicate is evaluated, it can be determined which formulas are now known to be satisfied and which formulas and conjunctions can be identified as unsatisfiable. This is then used to reduce the search space eliminating the unsatisfiable conjunctions and formulas. When eliminating formulas and conjunctions some of the so-far not yet evaluated predicates will no longer be referenced by the remaining conjunctions and these no longer need to be evaluated and can be skipped in the main loop.

This heuristic is based on the assumption that real-world ABAC policies are usually designed for a specific application domain and that target expressions of policies will share structure and predicates.

Definition 3.1. The following terms are the building blocks of the index and search algorithm:

- Let $s$ be a decision request. Let $S$ be the set of all possible decision requests.

- Let predicates $p: S \mapsto\{$ true, false $\}$ a function mapping a decision request to a Boolean value. Let $P$ be the set of all predicates occurring in the target expressions of policies in a policy retrieval point.

- Literals $l: S \mapsto\{$ true, false $\}$ are optionally negated predicates, i.e., for a $p \in P$ both $p$ and $\neg p$ are literals. Let $L$ be the set of all possible literals for predicates in $P$.

- A conjunction $c \in \mathcal{P}(L)$ is a subset of the available literals. A conjunction $c$ is satisfied for a request $s$, if and only if all literals evaluate to true for $s$. Let $C=\left(c_{1}, \ldots, c_{c}\right)$ an array of all conjunctions occurring in the formulas $F$.

- Let $F \subset \mathcal{P}(C)$ the set of formulas in disjunctive normal form, with minimal conjunctions representing all policy documents stored in the policy retrieval point. A formula $f$ is a subset of the available conjunctions and is satisfied when any conjunction is satisfied.

To efficiently identify elements to be eliminated the several index structures are precalculated.

For checking if a conjunction has been satisfied, for each conjunction, the number of literals in each conjunction is stored. The search algorithm will count how many literals of each conjunction are already satisfied and will compare with these numbers.

Definition 3.2. Let numberOf LiteralsInConjunction $=\left(l_{1}, \ldots, l_{c}\right)$ an array, where $l_{i}$ is the number of literals in $c_{i}, 1 \leq i \leq c$.

To identify which conjunctions are false given a single evaluated predicate, for each predicate the conjunctions which are evaluating to false for both possible values of the predicate are calculated in advance.

Definition 3.3. Let falseForTruePredicate $p \in \mathcal{P}(C)$ be the set of conjunctions which are false whenever $p(s)=$ true, $p \in P, s \in S$. Let falseForFalsePredicate $p \in \mathcal{P}(C)$ be the set of conjunctions which are false whenever $p(s)=$ false, $p \in P, s \in S$.

Whenever a conjunction is known to be true, all formulas containing the conjunction are satisfied and all other conjunctions of these formulas do no longer need to be evaluated if there are no other functions besides the ones containing the original conjunction. 
For each conjunction, the number of formulas is counted in which they are contained. This is used as a counter during search and is decremented each time such a formula is eliminated from the search space. This allows eliminating a conjunction as soon as this counter reaches zero.

Definition 3.4. Let numberOf FormulasWithConjunction $=$ $\left(f_{1} \ldots f_{c}\right)$, where $f_{i}$ is the number of formulas $f \in F$ where $c_{i} \in f$, $c_{i} \in C, 1 \leq i \leq c$.

Also, for each conjunction, it is identified which other conjunctions are in formulas containing it and in how many of these functions the conjunctions occur.

Definition 3.5. Let $F^{\prime}\left(c_{i}\right) \subseteq F$ be the set of formulas containing the conjunction $c_{i}$. Let $m\left(c_{i}\right)=(i, n) \in\{1, \ldots, c\} \times \mathbb{N}$ be a tuple of a conjunction index number and the number of formulas in $F\left(c_{i}\right)$ containing $c_{i}$. Let conjunctionsInFormulasReferencingConjunction $=$ $\left\{m\left(c_{i}\right)=(i, n) \mid 1 \leq i \leq c \wedge n \in \mathrm{N}\right\}$

Whenever a conjunction becomes satisfied, for each of these conjunctions, it can be counted how often a function containing them has been found. If all of the functions have been found containing them, they can be removed from further inspection for the remainder of a search.

To directly lookup conjunctions containing a predicate in its literals provide the sets organized in an array.

Definition 3.6. Let predicateInConjunction ${ }_{p} \in \mathcal{P}(C)$ be a set of conjunctions which contain $p \in P$.

Also an index for looking up formulas by predicate is calculated as an array.

Definition 3.7. Let predicateInFormula $a_{c} \in \mathcal{P}(F)$ be a set of formula containing $c \in C$.

These definitions are used to explicitly construct the given sets in memory upon startup of the PRP or whenever a change is made to the policies. These answer question a) of Section 2 on how to construct an index. No specific optimizations have been considered to make the index creation more efficient, as this is a rare operation compared to policy retrieval. With this index in place, search (i.e., policy retrieval) can be realized as described in Algorithm 1.

Algorithm 1 realizes the iterative count and eliminate approach. It is initialized by creating a set $C^{w}$ containing all conjunctions contained in $F$, the so-called candidate set and one empty set $C^{r}$, used to store all candidates that are satisfied, called result candidate set. Another two arrays for counting how many literals of any given conjunction is already true and how many formulas containing a given conjunction are already eliminated. An empty result set $R$ is defined. A formula or conjunction is eliminated, if the known predicates make it logically impossible for them to be satisfied, regardless of what the values of the remaining predicates may be.

For each predicate $p$, the algorithm checks, if it is referenced by any conjunction in the candidate set $C^{w}$. If not, the next predicate is inspected.

Then, it collects all conjunctions $C^{s}$ which are satisfied by $p$ and the previously evaluated predicates. These satisfied conjunctions are then added to the result candidate set.

Next, it calculates all conjunctions $C^{u}$ which are known to be false given $p(s)$ and the previously evaluated predicates.

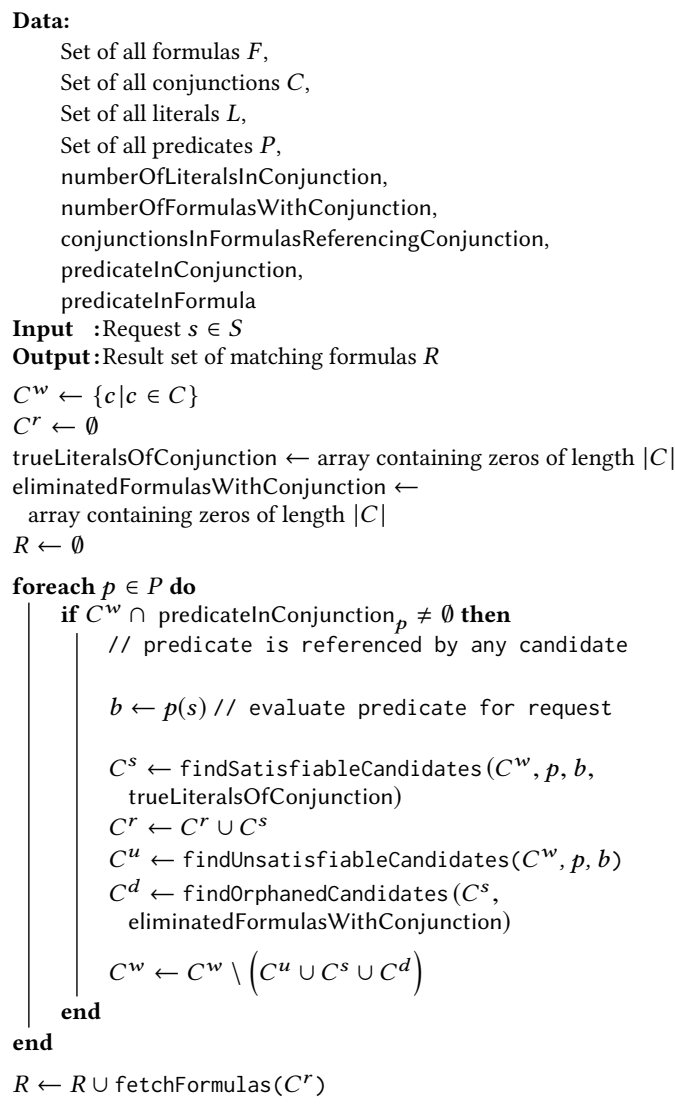

Algorithm 1: Count and Eliminate Search Algorithm

Finally, the conjunctions $C^{d}$ are calculated, which only appear in the functions containing conjunctions of $C^{s}$. As these functions have already been found, these conjunctions and matching predicates do no longer have to be inspected in the future.

All three sets $C^{s}, C^{u}$ and $C^{d}$ are then removed from the candidate set $C^{w}$.

The returned result set is determined by fetching all formulas containing a candidate from the result candidate set.

Finding the satisfied conjunctions implemented by counting. Since conjunctions may not contain multiple instances of the same predicate, the evaluation of a conjunction can be reduced to the number of successfully evaluated literals it contains. This is achieved by incrementing trueLiteralsOfConjunction. If the number of positive literal evaluations of a single conjunction is equal to its number of literals, stored in numberOf LiteralsInConjunction, the conjunction evaluates to true. To get the number of possibly satisfiable conjunctions, we use findUnsatisfiableCandidates with the negated predicate result.

As satisfied conjunctions may be part of multiple policies, the now evaluated policies are aggregated using the predicateInFormula structure.

Unsatisfied candidates are all conjunctions, in which the result of at least one literal is evaluated to be false and thus the conjunction can no longer be evaluated to true. 


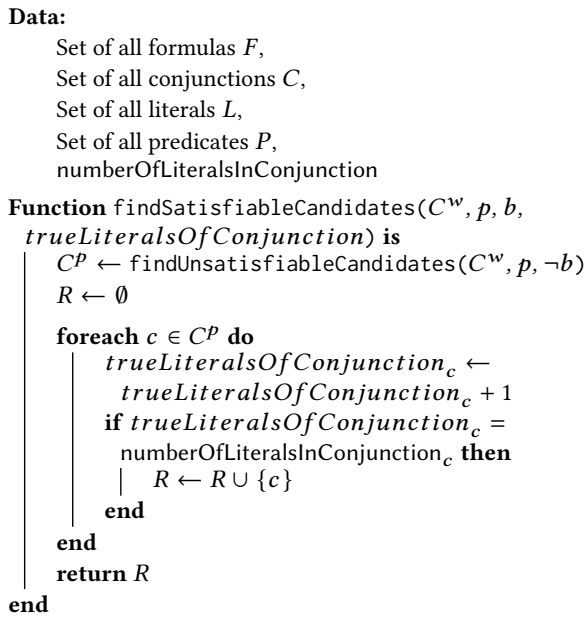

Algorithm 2: Find Satisfiable Candidates

Data:

Set of all formulas $F$,

Set of all conjunctions $C$,

Set of all literals $L$,

Set of all predicates $P$, predicatelnFormula

Function fetchFormulas $\left(C^{s}\right)$ is

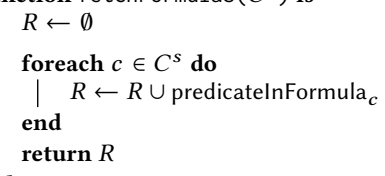

Algorithm 3: Fetch Formulas

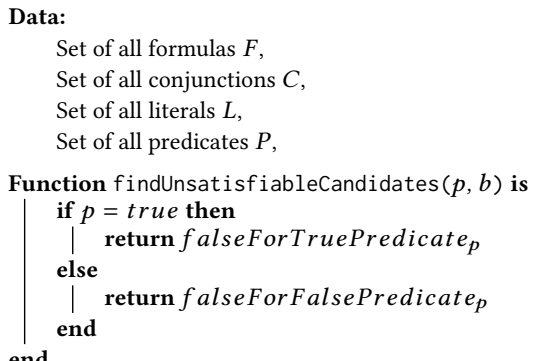

Algorithm 4: Find Unsatisfiable Candidates

Matching a policy may lead to a reduction of the candidates, if the policy contains more than one conjunction and those additional, not yet matched, conjunction share no further reference in additional policies. To resolve those orphans, the same reference counting technique is used, as in findSatisfiableCandidates. The structure eliminatedFormulasWithConjunction contains the current number of actively referenced formulas, for each conjunction. To navigate between the solved conjunction and the orphans, the index structure conjunctionsInFormulasReferencingConjunction is used. For every conjunction, all affected other conjunctions

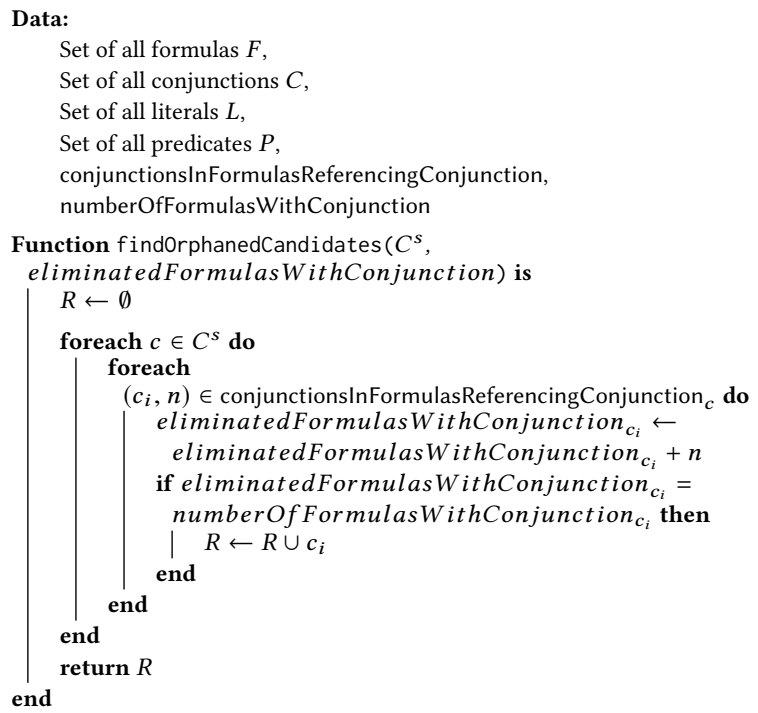

Algorithm 5: Find Orphaned Candidates

(contained in other policies) are saved, together with the number of occurrences of the particular conjunction through all formulas. This way, no additional loop over affected formulas is required, as this number can immediately be used to be added to eliminatedFormulasWithConjunction. Once a conjunction hits the threshold stored in conjunctionsInFormulasReferencing-

Conjunction, the conjunction is no longer been used in any not yet discarded policy.

The algorithm is the answer to question b) of Section 2 on how to use the index. The next sections will prove the correctness of the algorithm and examine its runtime.

\subsection{Correctness}

The correctness of the algorithm 1 in proven in two steps. First, it is shown that the algorithm without elimination of conjunctions finds all matching formulas and terminates. Second, it is shown that the elimination steps do not affect the result.

Algorithm 6 only uses a subset of the proposed functions and states no methods of reducing the number of candidates.

First, consider isReferenced. The condition of the if-clause evaluates an intersection between the current set of candidates and a set of preselected conjunctions containing the given predicate. Preconditions are: the data structure, the sets, and parameters have to conform to their respective definitions. The postcondition is, that it is only true when the current predicate is part of at least one conjunction in the candidate list. As predicateInConjuction is defined as the set of all conjunctions containing $p$ this holds.

The function findSatisfiableCandidates of Algorithm 2 uses findUnsatis fiableCandidates, to count the current evaluation status of every conjunction not rendered false. Our preconditions are the correctness of findUnsatisfiableCandidates and the conformity of all sets and the index numberOfLiteralsInConjunction to their definitions. Since in our model, a conjunction consists of a set of literals, no predicate can appear more than once in a single conjunction instance. As the algorithm 6 


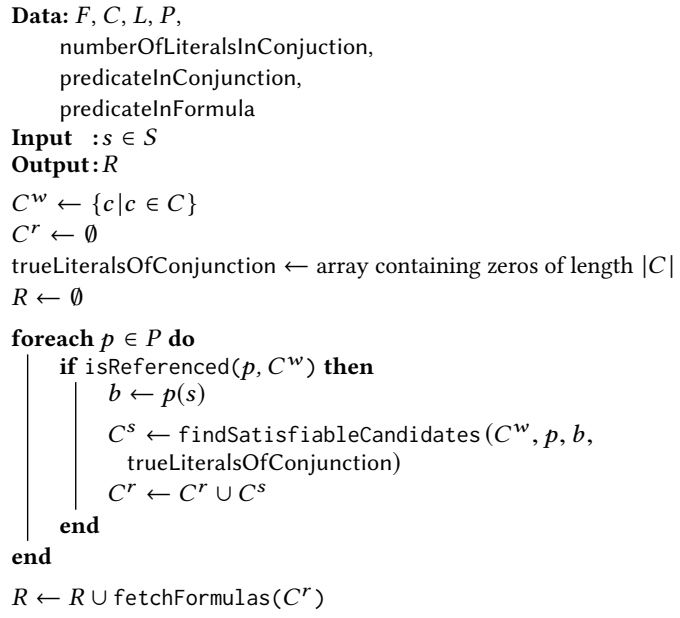

Algorithm 6: Simplified Count and Eliminate Search Algorithm

iterates over every predicate only once. This property can be used to memorize the current number of already positively evaluated literals within a conjunction and compare it with a reference of the conjunctions length. Since numberOfLiteralsInConjunction is defined as the number of literals within a conjunction term and findUnsatisfiableCandidates is correct, the number of $c \in$ $C^{p}$ added to trueLiterals $O f$ Conjunction for each conjunction can never exceed the respective entry within numberOfLiteralsInConjunction, as that implies all evaluations are done and the conjunction evaluates to true. This invariant holds true for all iterations, as $C^{p} \subseteq C$. From precondition and invariant postcondition can be concluded. During the evaluation of algorithm 6, every predicate is only checked once and the progress is stored in the runtime structure trueLiteralsOfConjunction. Every conjunction returned solves for true for a request.

As algorithm 3 fetchFormulas only uses a prepared index structure, its correctness is already given by its preconditions, which are the definitions of the sets and the structure predicateInFormula. Every conjunction matching belongs to at least one policy. Since every conjunction may only be solved once during the execution of algorithm 6, every policy returned is solved, as they represent formulas in DNF.

Given its subroutines are correct, we can now conclude the correctness of algorithm 6 . Preconditions are set and matching the definitions. For every predicate $p \in P$, the algorithm iterates of the set of candidates, initially $C^{w}=C$. Since no further operation is done on $C^{w}$, every call of isReferenced evaluates to true. Every single predicate is evaluated and the corresponding calls to findSatis fiableCandidates returns matching candidates, which in turn are mapped to their corresponding policies. Since no recursion takes place, and all sets are finite, algorithm 6 and the named functions terminate within a finite number of cycles.

When expanding the algorithm by adding the reduction of the candidates in every cycle by set of now successfully satisfied conjunctions. Pre- and postconditions do not change. Since we have shown isReferenced to be correct, only predicates not referenced in the list of candidates are skipped. Since they do not affect the set of conjunctions, evaluations of them add no value to solving formulas. This can be seen as an invariant condition. Removing unnecessary conjunctions from the working set does not alter the result set and does not contribute to further cycles. The algorithm still terminates.

By adding findUnsatisfiableCandidates and findOrphanedCandidates the original definition of Algorithm 1 is reached. This reduces the working set of candidates in each step.

The function findUnsatisfiableCandidates as defined in algorithm 4 correct by definition of the used index structures falseForTruePredicate and falseForFalsePredicate associate every predicate with their respective contribution in their conjunctions. As the definitions apply, the function is correct and contributes to the number of removable candidates. Since every candidate in the result set cannot be solved to true, they can be safely removed from the set.

Finally, examine findOrphanedCandidates as defined in Algorithm 5. Satisfying a policy allows to reduce the number of candidates by no longer reference, or orphaned, candidates. To compute the number of missing references, the working data set eliminatedFormulasWithConjunction, the index structure conjunctionsInFormulasReferencingConjunction and numberOfFormulasWithConjunction are used. Preconditions are, that all sets and structures comply with their respective definitions and no calls with the same parameters are made. Iterating over the number of successfully matched conjunctions, the index conjunctionsInFormulasReferencingConjunction is used to get all affected conjunctions and the number of policies referencing $c^{s}$. Adding this value to the corresponding entries in eliminatedFormulasWithConjunction documents the count of now no longer living references to these conjunctions (the number of target statements evaluated to true). After each call of Algorithm 1 the structures eliminatedFormulasWithConjunction and numberOfFormulasWithConjunction will be equal for all entries, thus proving the correctness of this function and concluding the correctness of algorithm 1.

\subsection{Runtime Analysis}

Estimating the average runtime of the search algorithm is depending on a good model of the input data. No such model is available. Thus, this paper resorts to estimating the worst-case runtime of the algorithm.

The outer loop of the algorithm iterates over the set of all predicates. The worst-case here is that no predicate can be eliminated. This may happen if all functions have exactly one and pairwise distinct predicates. Thus, the outer loop may have $|P|$ iterations.

Let $\phi(p)$ the runtime for evaluating the predicate $p$ without further knowledge about the complexity of the predicate evaluation. As the algorithm may at most evaluate each predicate once $\Phi_{P}=\sum_{p \in P} \phi(p)$ is the worst-case runtime.

The function findSatisfiableCandidates executes findUnsatisfiableCandidates which is constant time access to an existing index structure. As no modifications are made the assignment of $C^{p}$ can be made by reference in constant time. In the worst-case $C^{p}$ contains all conjunctions. As all operations inside 
the loop can be executed in constant time, the overall runtime of the function is $O(|C|)$.

Fetching the formulas and adding them to the result set can be executed in $O(1)$.

The function findUnsatisfiableCandidates is a direct index structure access in $O(1)$.

The elimination set operations at the end of the loop may in the worst-case, depending on the set implementation, take $O(|C|)$.

Ignoring the identification of orphan conjunctions for now, overall the runtime is $O(|P||C|)+\Phi_{P}$.

The function findOrphrandCandidates takes the currently identified satisfied conjunctions as an input and iterates over them in its outer loop. As any conjunction is found to be satisfied at most once, during the runtime of the algorithm, the inner loop is executed at most $|C|$ times overall iterations of the main algorithm. The inner loop iterates over a list conjunctions, where each conjunction appears at most once, and thus the inner operations are executed at most $|C|$ times. As these operations can be executed in $O(1)$ the runtime of the orphan identification is $O\left(|C|^{2}\right)$.

Thus, the overall worst-case runtime of the search algorithm is $O\left(|P||C|+|C|^{2}\right)+\Phi_{P}$.

Assuming a naive implementation of the search algorithm, evaluating all functions one after the other while caching the results of evaluating predicates. In this algorithm, the measure $|C|$ is not natural, as it does not examine if conjunctions occur multiple times across different functions. However, in the worst-case, all functions contain all conjunctions. Thus, evaluating a single function may cost, ignoring predicate evaluation, $O(|P||C|)$. Together for all functions and taking into account predicate evaluation, this amounts to $O(|F||P||C|)+\Phi_{P}$. This means, that depending on $|C|$ and $|F|$ of the given concrete datasets it cannot be generally be said that one is always better in the worst-case.

\subsection{Predicate Order}

An important aspect of the algorithm is the order in which the main loop evaluates the individual predicates. Depending on the specific functions contained in the search space this order has a significant impact on the speed-up compared to a naive search implementation with caching. Determining the optimal order is an additional optimization problem, which is similar to well studied variable ordering problem for Binary Decision Diagrams, e.g., [1, 17] This section proposes an initial heuristic for ordering predicates based on a few key properties of the predicates, with a specific look at SAPL policies. Similar heuristics will apply for XACML.

Different predicates will change which sets of conjunctions are eliminated in each step. Thus, before constructing the index structure a predicate order should be identified which maximizes the probability of eliminating as many conjunctions as early as possible at runtime. For any given set of policies, many optimal orderings may exist. Identifying such an ordering equates to a search in the space of all permutations.

The first observation to make is in how many formulas an individual predicate occurs in. Such a predicate has the potential to discover several matching functions at once. These are then removed from the search and will potentially eliminate a significant amount of conjunctions from the search as they may no longer be referenced by any function, i.e., they become orphans and are removed and reduce the number of conjunctions to be inspected each iteration.

Definition 3.8. The number of formulas using the predicate $p$ is defined as:

$$
\text { funCount }_{F}: P \rightarrow \mathbb{N}, p \mapsto \mid\{f \in F \mid f \text { contains a literal of } p\} \mid
$$

The second observation is how often a predicate $p$ is used in positive or negative literals. If the use of $p$ is distributed about equally between these, each evaluation of $p$ guarantees that about half of the conjunctions containing $p$ are removed from the candidate set, independently of the evaluation result of $p$. A predicate only occurring in positive literals way eliminate all conjunctions at once, but it also may not eliminate any. Thus the guarantee for elimination is preferable.

Definition 3.9. Count positive and negative occurrences of the literal. The number formulas using the predicate $p$ in a positive literal is:

$$
\text { funCount }_{F}^{+}: P \rightarrow \mathbb{N}, p \mapsto \mid\{f \in F \mid f \text { has a positive literal of } p\} \mid
$$

The number formulas using the predicate $p$ in a negating literal is:

$$
\text { funCount }_{F}^{-}: P \rightarrow \mathbb{N}, p \mapsto \mid\{f \in F \mid f \text { has a negative literal of } p\} \mid
$$

The relation between positive and negative occurrences of literals of the predicate $p$ across the functions is:

$$
\text { split }_{C}: P \rightarrow \mathbb{Q}, p \mapsto \frac{\text { funCount }_{F}^{+}(p)-\text { funCount }_{F}^{-}(p)}{\text { funCount }_{F}^{+}(p)+\text { funCount }_{F}^{-}(p)}
$$

The third observation is, that if a conjunction is of length one, only this one literal is required to satisfy it, identify a matching function, and eliminate the related orphan conjunctions. Thus, an attempt should be made to prioritize the elimination of short conjunctions. This is done by assigning those predicates a priority, which are on average contained in short conjunctions. Or stating this differently prioritize predicated contributing the most on average to fulfilling conjunctions. Where the contribution of each predicate in a conjunction $c$ is $\frac{1}{|c|}$.

Definition 3.10. Let $C^{\prime}(p) \subseteq C$ the set of conjunctions containing literals with $p$.

$$
\text { Let } \text { contrib }_{F}: P \rightarrow \mathbb{Q}, p \mapsto \sum_{c \in C^{\prime}(p)} \frac{1}{|c|\left|C^{\prime}(p)\right|}
$$

The final observation is, that in the evaluation of predicates does not occur in constant time. It depends on both, the underlying policy language expressions and the request. For example, checking if a coordinate is contained in a polygon for geofencing is significantly more costly than a comparison of numbers. Costly predicate evaluations should be delayed providing a chance, that other predicate values already eliminate the need for evaluating the costly predicate. Thus, for each predicate, a cost value should be assigned. Determining this value is not obvious. Either the policy engine developers provide some estimates for different operations which are aggregated over the expression, or the index starts with no knowledge of the cost and profiles the runtime of different predicates across policy retrieval for subsequent requests and learns the runtime cost. 
Definition 3.11. Let cost $P \rightarrow \mathbb{N}\{0\}$ the cost map for all predicates.

This map may be derived in different ways. E.g., through static code analysis or measurements at runtime. If no knowledge about the cost of predicates is available $\operatorname{cost}(p)$ shall be 1 for all $p$.

For now, it is not clear how strong each metric impacts the early elimination of conjunctions and formulas. The following definition of a priority is based on intuitively assumed impact. This is just a first attempt and further research is required. Also, machine learning approaches may be useful to determine a good function for calculating the priority. For evaluating the search algorithm the following definition is used:

$$
\begin{aligned}
& \text { Definition 3.12. Let priority }_{(F, C)}: P \rightarrow \mathbb{Q}, p \mapsto \\
& \frac{\text { funCount }_{F}(p)^{2} \cdot\left(2-\operatorname{split}_{C}(p)^{2}\right) \cdot \operatorname{contrib}_{F}(p)^{\left(2-\operatorname{contrib}_{F}(p)\right)}}{\operatorname{cost}(p)}
\end{aligned}
$$

During index construction, these priorities are calculated and the predicate array is sorted accordingly in ascending priority. Overall this is an initial pragmatic ad-hoc approach to answer question c) of Section 2 based on the presented observations.

\section{EVALUATION}

The fundamental hypothesis motivating the heuristic implemented by the algorithm, is that shared structure and predicates in the target expressions can be leveraged to improve the performance of policy retrieval.

Therefore, two generators for test data were implemented. The first generator and benchmark generated a set of policies and target expressions with no real-world use-case in mind, this version will be referred to as "Fully Random Policy Generation". As an explicit worst-case scenario is yet unknown, this case has been constructed to be as close to a worst-case scenario for the policy index, as target expressions share little structure.

The second generator and benchmark however are based on patterns found in a real-world medical scenario, which are then used to scale up the problem randomly to examine the behavior of the algorithm with very large data sets. This version will be referred to as "Structured Random Policy Generation".

These benchmarks do only examine the policy retrieval of the decision process and not the complete policy evaluation. Thus, the generated rules beyond the target expressions illustrate the real-world scenario but have no impact on the policy retrieval.

To ensure transparency and reproducibility, both benchmark implementations have been published on GitHub [6], [5].

4.0.1 Fully Random Policy Generation. This algorithm generates fully random target expressions. It is parameterized by the chances of conjunction, disjunction, and negation as well as parentheses, resulting in complex non-natural target statements.

The following listing shows an example of a policy, consisting of 5 variables from a pool of 1000 .

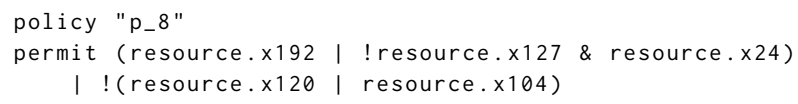

Listing 1: SAPL policy randomly generated
The resulting policy documents are then matched against randomly generated decision requests. The requests are created using the same variable pool, with each variable having a previously specified chance of being set to "true".

4.0.2 Structured Random Policy Generation. The starting point for the implementation of a structured policy generation process was a set of policies written by a human for a fictional application in a hospital environment.

The base for the policy generation model is the observation, that for every environment there is a set of subjects with different positions, interacting with various resources. The amount of subjects interacting with a resource is different for every resource. The type of access can furthermore be grouped by the position of a subject.

The policies are written for the real-world scenario, more precisely the target expressions, were then analyzed to identify characteristics of the test data. This made it possible to put in variables that can be used to scale the process to any desired size.

The listing 2 shows an example policy generated using the new approach.

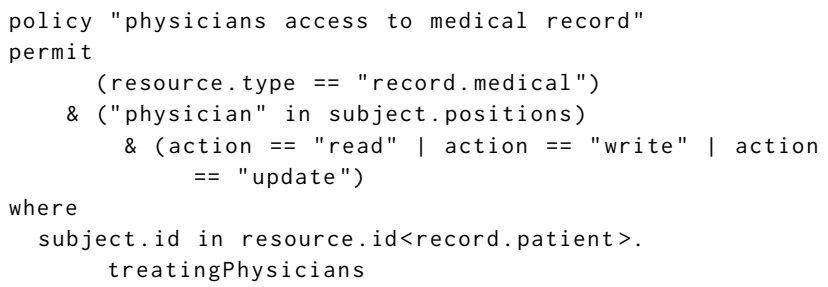

Listing 2: Sample SAPL policy generated using structured approach

Subjects. The first parameter for the generation process is the number of subjects in the domain. Another parameter of the process is the maximum amount of positions a single subject can have. Observations led to the assumption that the majority of subjects has no more than different 5 positions in a domain. The actual amount of positions of a subject will be randomized by the generation process.

The analysis of the policies written for the hospital domain exposed that a large set of policies exists, that concern the individual subject itself and not its position. For a predefined percentage of subjects, the process will generate these subject-specific policies.

One example would be a subject for which all permissions have been withdrawn and therefore all actions in the domain are forbidden.

Positions. Our policy generation model differentiates between two types of positions, with both of them requiring different kinds of policies.

- General access positions have permissions that are universally applicable (for all resources)

an example of a general position would be a backup system having full access to all resources without any limitations.

- Specific access positions have permissions that are specific to a resource 
These two types can be further divided by the number of actions permitted. 3 categories of access were identified and used in the model:

- permitted to perform all available actions (Full Access)

- read-only permission (Read Access)

- a specific subset of actions is permitted (Custom Access)

For each of the resulting sub-categories, different policies will be generated reflecting the respective access scheme. The generation process accepts a likelihood for each type to be used for a position in the domain. This parameter can be used to shift the shares between the categories.

Positions granted with permissions that are agnostic to resources (General Access) are uncommon. It turned out to be more likely that a position is only granted access to a defined subset of resources in the domain (Specific Access). The analysis of the policies in the hospital application confirmed this assumption, which is why the policy generation tool favors creating policies that are linked to a specific resource.

Resources. Similar to positions, resources can be split into groups. Inside a domain, resources exist that can be accessed by any subject, regardless of its position. We will refer to these as unrestricted resources.

For restricted resources the algorithm produces multiple policies, reflecting different access schemes on the resource. The generation algorithm uses given probabilities to select one of the following access schemes for every position in the domain.

- Full (all actions)

- Read (read-only)

- Custom (a custom subset of actions) A random subset of actions is generated

- None

The position is ignored and no policy is generated

Looking at the hospital domain again, showed that the percentage of restricted, position-specific resources is much higher than for unrestricted resources.

Actions. Besides subjects, positions, and resources the overall number of available actions on resources is an important part of the model. Having full access to a resource is equal to permitting all defined actions. For policies reflecting a full access schema, the enumeration of actions is omitted and therefore the target expression contains fewer variables.

When the number of overall actions inside the domain increases it becomes more likely that each policy has a different set of actions expressed in its target statement. This has a direct impact on the number of policies the algorithm can eliminate by evaluating a single variable.

Extensions. For certain combinations of positions and resources, the access must be further detailed, requiring additional policies to be generated. These extended policies are also part of the policy generation model and contain at least one of the following additional sections:

- Body

Extends the condition in the target expression and further limits the policy applicability
- Obligation

Tasks that must be executed before granting access

- Advice

Similar to obligation, but the failure of execution does not prevent access

- Transformation

Transform resource for access

Although these extensions are not part of the policie's target expression, the generation algorithm must create an additional policy for the position/resource-combination. The result is multiple policies sharing the same target expression which then affects the policy indexing strategy.

Resulting Policies. The structured generation process is creating separate policies for every position accessing a resource. For positions sharing the same access scheme on a resource, a single policy containing all positions is created. An example of this would be all positions having only read access to a resource.

4.0.3 Decision Request Generation. To generate requests similar to a real-world use-case, the benchmark tool uses the previously generated policies as input to create meaningful requests. This is done by parsing all generated policies and extracting attributes used by the target expressions. These are added randomly to the decision requests.

In practice, errors will occur naturally, having an impact on the policy engine. This has been considered for the implementation of the generation tool, which will therefore also generate invalid requests to cause errors. Requests that are completely unrelated to the policies are also generated, forcing the PRP to analyze as many documents as possible before returning a result.

With the above considerations taken into account, the updated benchmark implementation is now generating realistic decision requests.

\subsection{Technical Considerations}

The algorithm and benchmark were implemented as part of featurecomplete open source policy engine implementation for SAPL [Blind Reference]. The engine and benchmark were implemented using Java and it was taken care to make use of efficient data structures like arrays, bit sets, and Guava immutable collections where applicable.

All benchmarks were executed within the same environment. The system was a macOS Catalina (10.15.6) machine, using an Intel Core i7-9750H CPU (base clock speed of $2.6 \mathrm{GHz}, 6$ cores) with 16 GB DDR4 RAM. The Java version used to build and run the benchmark is "AdoptOpenJDK build 15+36)" running in a 64bit VM. All iterations of the benchmark were executed with the same heap configuration: -Xms4096m -Xmx14000m

Due to the nature of Java, certain inaccuracies can not fully be eliminated. Garbage collection or growing memory allocation are JVM-specific conditions that need to be taken into account.

Since micro-benchmarking is furthermore prone to error, multiple runs of a benchmark configuration were performed. To make any comparison possible we used the average and median of all runs. 


\subsection{Benchmark Results}

The benchmarks presented in this section are measuring the time the index takes to retrieve all matching policy documents to a given decision request, comparing the performance of the algorithm on the two data sets against a base-line implementation. No implementation of other policy indexing algorithms was available. As the XACML standard does not detail database query creation for policy indexing and only offers a naive approach as an alternative, the latter was selected as the baseline for comparison. This naive algorithm sequentially iterates over all documents stored in the $\mathrm{PRP}$, matching their target expression against the decision request.

Benchmarks Fully Random. The fully random policies serve as a test of how the algorithms would perform in a close to a worst-case scenario. Comparing it with the structured approach should help to validate the correctness of the basic hypothesis that structure and shared predicates will help the algorithm to perform significantly better.

Twelve test cases have been defined for the random policy generator. Ten test cases were defined to evaluate the runtime behavior in growing numbers of policies and predicates. Additional two cases were written to evaluate the impact of different conjunction- \& disjunction-ratios.

Each test case has three parameters:

- number of policies generated

- the number of predicates in every target expression

- total number of predicates available from which these are selected

Every test case was executed 100 times and the median and average of the execution times was calculated. The average and median execution times are shown in Figure 1. For the first ten cases, chances of conjunction were set to $90 \%$, negation to $30 \%$ and opening brackets to $20 \%$. The remaining two consists of 1000 policies, each consisting of ten variables coming from a variable pool with the size of 1000. The test case MH Conj. Max TC uses a conjunction chance of $70 \%$, whereas ML Conj. Max TC has chance for conjunction of only $30 \%$. It can be observed that the indexed version outperforms the naive implementation throughout all test cases. An issue with this benchmark are the small policy counts of these tests. The execution times of both implementations are below $20 \mathrm{~ms}$, which makes it difficult to conclude.

In Figure 2 it is easier to compare the results. In this chart, the percentage of the speed-gain using the indexing algorithm compared to the naive implementation is shown. The average of all benchmark configurations is represented as a dotted line.

In summary, the algorithm introduced in this paper is around $85 \%$ faster than the naive implementation. Considering that this benchmark was designed to be especially challenging for the algorithm, this significant gain is already very promising for practical application.

Benchmark Structured Random. Six different benchmark configurations were defined and executed using the structured strategy. Comparing the setup to the previous benchmarks, it can be noticed that the size of the policy sets is much bigger for the last two test cases.

The following parameters were used to generate the policies:

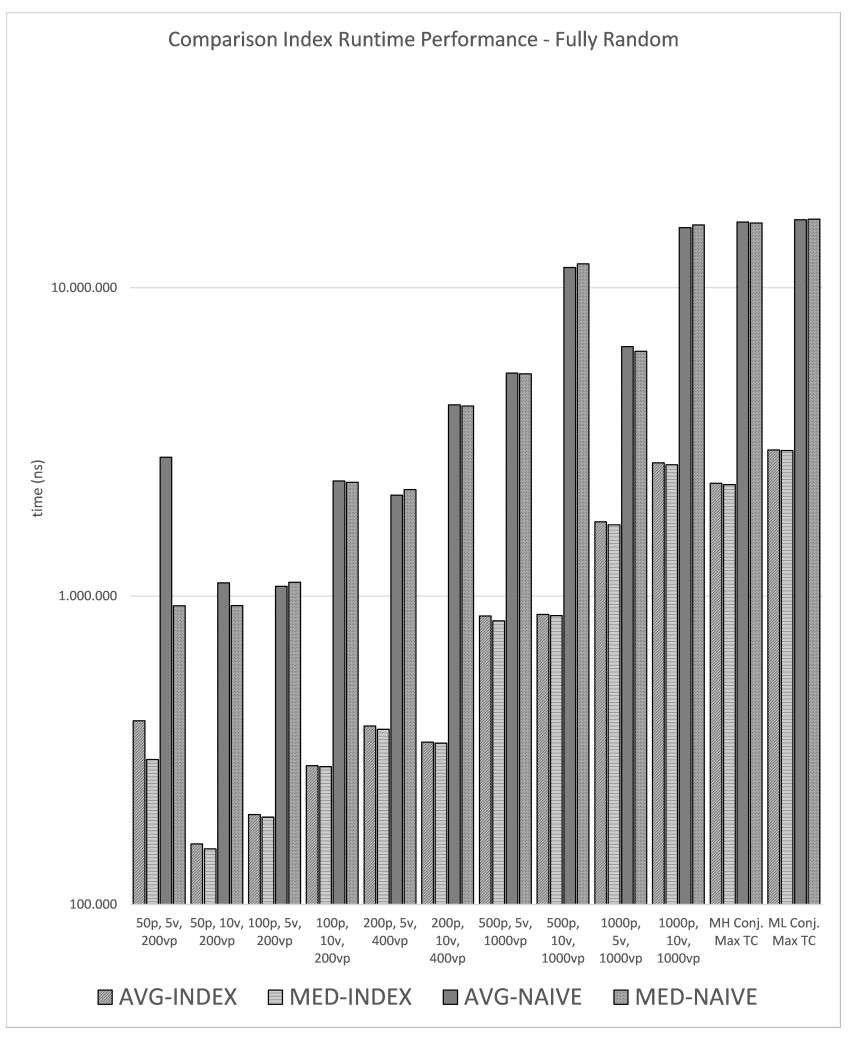

Figure 1: Comparison of Index Runtime Performance - Fully Random

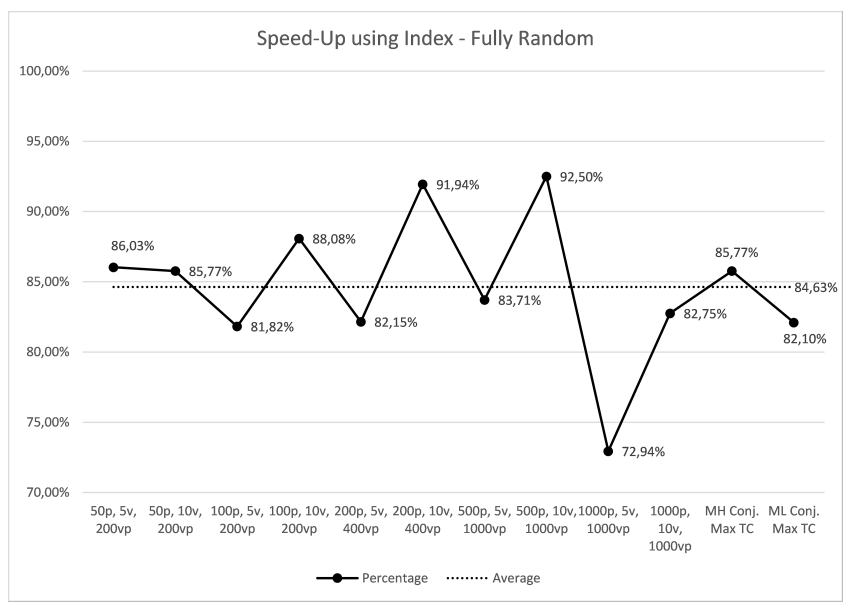

Figure 2: Percentage of Naive Runtime - Fully Random

- 50p, 500vp

10 subjects, 3 positions, 20 resources, 15 actions

55 policies were generated

- 100p, 1000vp

15 subjects, 5 positions, 25 resources, 15 actions

106 policies were generated

- 550p, 10.000vp 


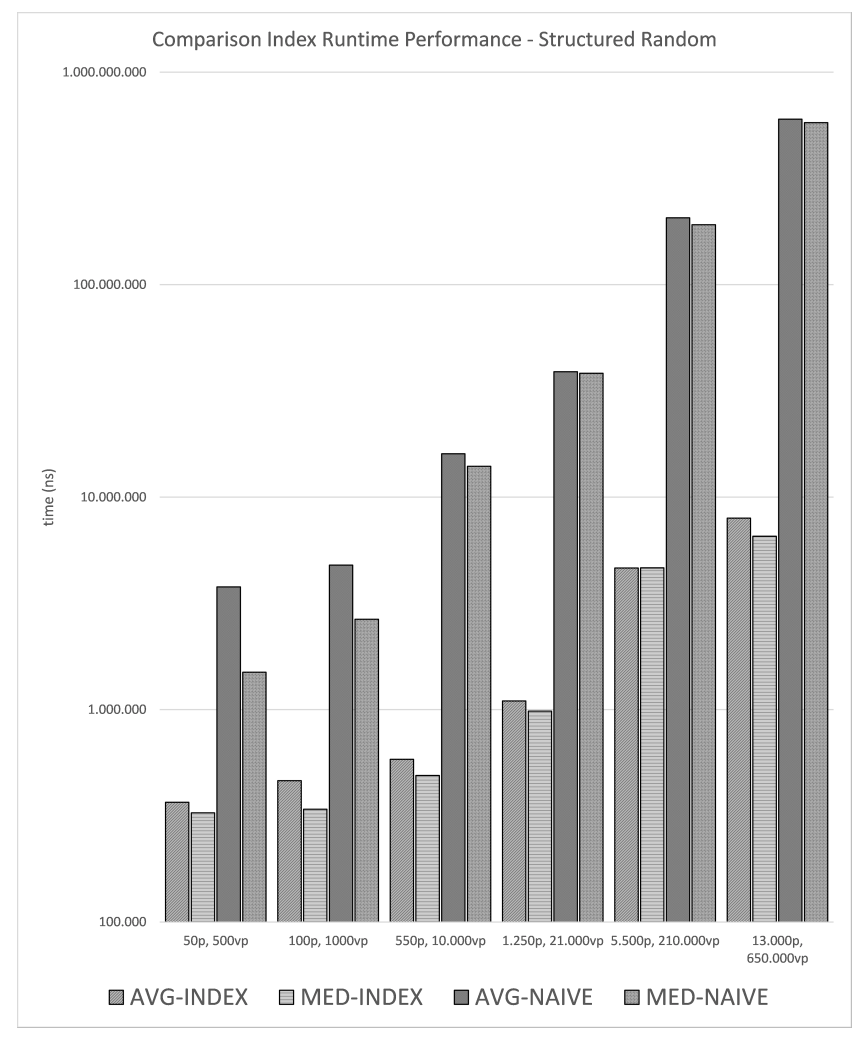

Figure 3: Comparison of Index Runtime Performance Structured Random

50 subjects, 10 positions, 80 resources, 30 actions 580 policies were generated

- $1.250 \mathrm{p}, 21.000 \mathrm{vp}$

100 subjects, 20 positions, 100 resources, 30 actions

1.264 policies were generated

- $5.500 \mathrm{p}, 210.000 \mathrm{vp}$

100 subjects, 30 positions, 300 resources, 150 actions

5.615 policies were generated

- $13.000 \mathrm{p}, 650.000 \mathrm{vp}$

250 subjects, 50 positions, 500 resources, 250 actions

13.234 policies were generated

For the labels in used in figures 3 and 4 rounded policy and variable numbers are used.

Figure 3 shows the results of the benchmark executions. Again every benchmark was executed 100 times and the median and average execution time was calculated. Starting with a small set, consisting of only 50 policies, the number increases up to 15.000 policies for the last test case.

The Y-axis has a logarithmic scale with base 10 , as the time difference between the two implementations was bigger with the increasing size of the policy sets. Comparing the execution time of the last benchmark, the naive implementation took $600 \mathrm{~ms}$ on average while the indexed version returned the matching policies in around $8 \mathrm{~ms}$.

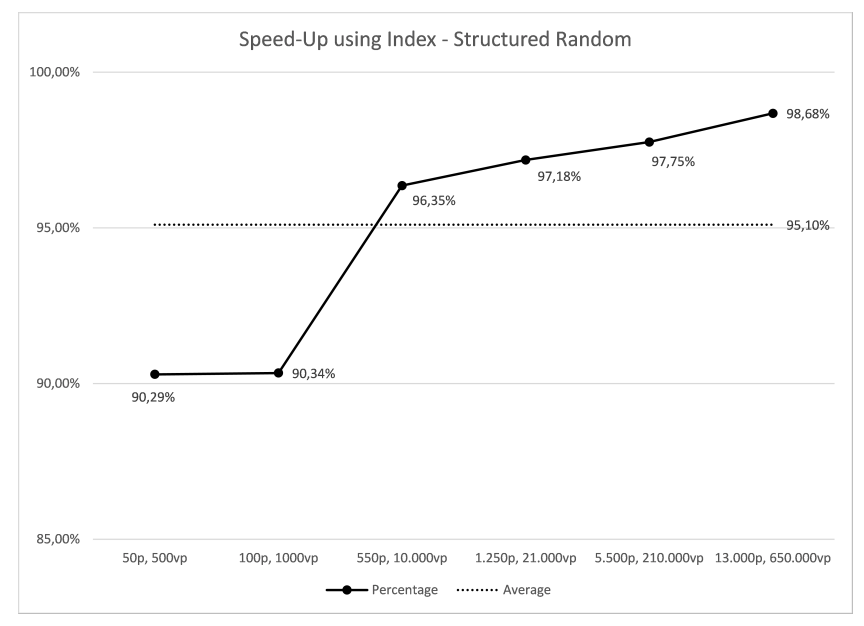

Figure 4: Percentage of Naive Runtime - Structured Random

To make the results more tangible, Figure 4 also shows the percentage of the speed-gain using the indexing algorithm compared to the naive approach.

Looking at all the numbers of all benchmarks, the presented algorithm within a Policy Retrieval Point is up to $98 \%$ faster than the naive approach.

Summary. The benchmarks answer questions d) and e) of the Section 2. It is evident, that the count and eliminate algorithms significantly outperform the naive approach for both random and semi-structured data. Here, the more structured data set again yields an increased performance gain. This also validates the hypothesis stated as the basis of the algorithm, that predicates and structure shared by policies can be leveraged in policy retrieval.

\section{CONCLUSIONS}

This paper presented and evaluated a novel index and search algorithm for retrieving policy documents from a PRP. The empirical studies have shown that by employing the index PRP performance can be increased significantly in most cases, even for unstructured data sets. A remaining reason in favor of the naive strategy is the auditing of policy decisions, as logging the calculations made by the naive approach leads to information that is easy to understand by a human auditor.

While the implementation of the algorithm and its evaluation has been conducted for SAPL it is also applicable to XACML. It is likely to be easier to implement for XACML, as the target elements are already normalized and the predicates are generally simpler than in SAPL.

Overall the algorithm is solving the general problem of searching for Boolean functions over a set of predicates. Thus, it may have even wider applications outside of ABAC.

The significant performance gains in policy retrieval confront authors of policies with some interesting trade-off considerations when writing policies. In theory, any rule within a policy that is not requiring IO operations to access an external policy information point could be rewritten as part of the target expression, delegating more reasoning from PDP to PRP where the index can potentially 
speed-up the overall decision-making process. If done consequently, the remaining rules would only be required for reasoning over external request-time attributes. This however may result in less well-structured documents that are harder to write and understand by humans.

\section{FUTURE WORK}

The final trade-off mentioned in the last section also indicates the potential for automated policy optimization, so before publishing a policy to the PRP, the policies could be optimized and automatically rewritten into equivalent policies maximizing the number of rules expressed in the target element. This could retain the ease of authoring of policies. However, reasoning about and debugging decisions at runtime may become more difficult.

Selecting the predicate order potentially has a major impact on lookup times. The presented heuristic for selecting a matching predicate order is a first attempt that can be calculated quickly. It has the major advantage that the variable order may be optimized at runtime without the need to completely reconstruct the index. It would be sufficient to alter the sequence in which predicates are evaluated. One approach to examine is to take measurements about the effectiveness of reducing the number of candidate conjunctions or to measure the actual cost of evaluating the predicates. This should also be compared to other existing heuristics for optimizing permutations.

Further experiments should be conducted using larger-scale sets of policies and requests from real-world applications to evaluate the impact of policy indexing algorithm on the overall decision-making process of a PDP under load.

So far, no comparative studies with other indexing approaches for Boolean function have been conducted. Especially adopting the BE-Tree $[14,15]$ to support policy target elements beyond the predicates outlined in the original work may be feasible. It should be examined how these methodologies could be applied to the provided use case and comparative evaluations should be performed.

\section{ACKNOWLEDGMENTS}

This work has been carried out in the framework of the European Union's Horizon 2020 esearch and innovation program under grant agreement No 957852 Virtual Power Plant for Interoperable and Smart isLANDS'- VPP4ISLANDS.

\section{REFERENCES}

[1] Beate Bollig, Martin Löbbing, and Ingo Wegener. 1995. Simulated annealing to improve variable orderings for OBDDs. In In Int'l Workshop on Logic Synth.
Citeseer.

[2] Françoise Fabret, H. Arno Jacobsen, François Llirbat, Joăo Pereira, Kenneth A. Ross, and Dennis Shasha. 2001. Filtering Algorithms and Implementation for Very Fast Publish/Subscribe Systems. SIGMOD Rec. 30, 2 (May 2001), 115-126. https://doi.org/10.1145/376284.375677

[3] Dominic Heutelbeck. 2019. Attribute Stream-Based Access Control (ASBAC) Functional Architecture and Patterns. In Proceedings of the 2019 International Conference of Security and Management (SAM'19) (2019-07-29).

[4] Dominic Heutelbeck. 2019. The Structure and Agency Policy Language (SAPL) for Attribute Stream-Based Access Control (ASBAC). In Proceedings of the ETAA 2019 : 2nd International Workshop on Emerging Technologies for Authorization and Authentication.

[5] Dominic Heutelbeck. 2021. SAPL Policy Engine Benchmarks Snapshot. https: //github.com/heutelbeck/sapl-demos/tree/sacmat2021

[6] Dominic Heutelbeck. 2021. SAPL Policy Engine Snapshot. https://github.com/ heutelbeck/sapl-policy-engine/tree/sacmat2021

[7] Vincent C. Hu, David Ferraiolo, Rick Kuhn, Adam Schnitzer, Kenneth Sandlin, Robert Miller, and Karen Scarfone. 2014. Guide to Attribute Based Access Control $(A B A C)$ Definition and Considerations. Technical Report. National Institute of Standards and Technology. https://doi.org/10.6028/nist.sp.800-162

[8] Donald C Latham. 1986. Department of defense trusted computer system evaluation criteria. Department of Defense (1986).

[9] Adam J Lee and Marianne Winslett. 2008. Towards an efficient and languageagnostic compliance checker for trust negotiation systems. In Proceedings of the 2008 ACM symposium on Information, computer and communications security. $228-239$.

[10] Alex X Liu, Fei Chen, JeeHyun Hwang, and Tao Xie. 2008. Xengine: a fast and scalable XACML policy evaluation engine. ACM SIGMETRICS Performance Evaluation Review 36, 1 (2008), 265-276.

[11] Peter Bro Miltersen, Jaikumar Radhakrishnan, and Ingo Wegener. 2005. On converting CNF to DNF. Theoretical Computer Science 347, 1-2 (2005), 325-335. https://doi.org/10.1016/j.tcs.2005.07.029

[12] Ronit Nath, Saptarshi Das, Shamik Sural, Jaideep Vaidya, and Vijay Atluri. 2019. PolTree: A Data Structure for Making Efficient Access Decisions in ABAC. In Proceedings of the 24th ACM Symposium on Access Control Models and Technologies. 25-35.

[13] Santiago Pina Ros, Mario Lischka, and Félix Gómez Mármol. 2012. Graph-based XACML Evaluation. In Proceedings of the 17th ACM Symposium on Access Control Models and Technologies (SACMAT '12). ACM, New York, NY, USA, 83-92. https: //doi.org/10.1145/2295136.2295153

[14] Mohammad Sadoghi and Hans-Arno Jacobsen. 2011. BE-tree: An Index Structure to Efficiently Match Boolean Expressions over High-dimensional Discrete Space. In Proceedings of the 2011 international conference on Management of data-SIGMOD '11 (SIGMOD '11). ACM, 637-648. https://doi.org/10.1145/1989323.1989390

[15] Mohammad Sadoghi and Hans-Arno Jacobsen. 2013. Analysis and optimization for boolean expression indexing. ACM Transactions on Database Systems 38, 2, Article 8 (2013), 47 pages. https://doi.org/10.1145/2487259.2487260

[16] Ravi S Sandhu. 1998. Role-based access control. In Advances in computers. Vol. 46. Elsevier, 237-286.

[17] Ingo Wegener. 2000. Branching programs and binary decision diagrams: theory and applications. SIAM.

[18] Steven Euijong Whang, Hector Garcia-Molina, Chad Brower, Jayavel Shanmugasundaram, Sergei Vassilvitskii, Erik Vee, and Ramana Yerneni. 2009. Indexing Boolean expressions. Proceedings of the VLDB Endowment 2, 1 (2009), 37-48. https://doi.org/10.14778/1687627.1687633

[19] XACML 3.0 Committee. 2013. eXtensible Access Control Markup Language (XACML) Version 3.0. http://docs.oasis-open.org/xacml/3.0/xacml-3.0-corespec-os-en.html accessed 2019-05-10.

[20] XACML 3.0 Committee. 2015. Abbreviated Language for Authorization Version 1.0. https://www.oasis-open.org/committees/download.php/55228/alfa-forxacml-v1.0-wd01.doc accessed 2019-05-10. 